\title{
Analysis of cyclic prefix length effect on ISI limitation in OFDM system over a Rayleigh-fading multipath
}

\author{
Sarah Zanafi, Noura Aknin \\ Information Technology and Modeling Systems Research Unit (TIMS), Abdelmalek Essaadi University, \\ Tetouan, Morroco
}

\begin{tabular}{l} 
Article Info \\
\hline Article history: \\
Received Jan 2, 2019 \\
Revised Nov 24, 2020 \\
Accepted Jan 13, 2021 \\
\hline Keywords: \\
AWGN \\
CP \\
ICI \\
ISI \\
OFDM \\
Rayleigh \\
\hline
\end{tabular}

\begin{abstract}
In this work, the influence of the cyclic prefix on the performance of the OFDM system is studied. We worked out an OFDM transceiver using a 16 QAM modulation scheme, a comparison of the BER for various lengths of the cyclic prefix has been achieved, and the influence of the noise introduced in the channel has been highlighted, for both a Gaussian and Rayleigh noise. The simulation was carried out on MATLAB where the curves of the BER for various lengths of the cyclic prefix are given and compared. We also adopted as a metric the QAM constellation to show the dispersion of the carriers as a consequence of the transmission channel, the mitigation of this effect by the $\mathrm{CP}$ is noticeable.
\end{abstract}

This is an open access article under the CC BY-SA license.

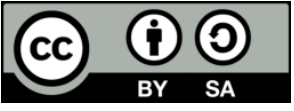

Corresponding Author:

Sarah Zanafi

Information Technology and Modeling Systems Research Unit (TIMS)

Abdelmalek Essaadi University

Faculty of science, Avenue de Sebta, Mhannech II, 93002 Tetouan-Morocco

Email: s.zanafi@gmail.com

\section{INTRODUCTION}

One of the major issues in telecommunication is to adapt the information to be passed over a channel to the channel characteristics [1]. For frequency selective channels, an efficient technique is to use a multi-carrier modulation in which the blocks of information are modulated by the Fourier transform [2]. This technique known as orthogonal frequency division multiplexing (OFDM), showed to be very efficient [3]. The information is transmitted on $\mathrm{N}$ different sub-carriers [4], every sub-carrier having an interval of time multiplied by $\mathrm{N}$ [5]. By assigning various sets of sub-carriers to various users, the transmitted carriers are orthogonal between them, Therefore allowing every user to make correspond his symbols of data to the corresponding sub-carriers. The system is $\mathrm{N}$ times more robust against the inter-symbol interference (ISI) whereas the global rate of transmission remains identical. The spectral efficiency of the OFDM modulation is thus excellent because sub-carriers can overlap. Nevertheless, the most difficult issue to deal with is the inter symbol interference (ISI), resulting from the multipath distribution of the signal on the transmission channel [6], the use of cyclic prefix in the emission allows to reduce the complexity of terminals through the use of FFT algorithm [7].

The mathematical model of the ISI can be obtained by performing a linear convolution of the impulse channel response with the bit stream in time domain [8]. The Inter carrier interference (ICI) reflects the loss of orthogonality between the carrier frequencies as a result of the channel frequency response [9]. The cyclic prefix reduces at the same time ISI and ICI by transforming the linear convolution into a discrete one by inserting the $\mathrm{CP}$ at the beginning of each block [10]. To this end, different ICI cancellation methods have been proposed in the literature, In [11] authors presented the multilevel soft frequency reuse (SFR) which aims to limit and manage the inter-cell interference, this method uses cell sectorization, in which each 
sector antenna covers a particular region of the cell, despite its efficiency in reduction ICI, this method decreases the capacity in the cell. Reducing each customer throughput implies a reduction in spectral efficiency. Some other studies and techniques like MMSE [12] have been introduced [13] to limit the effects of ICI and ISI, these approaches require a high complexity in their implementation, whether on transmitter or receiver side. In [14] a residues coding scheme is designed in order to measure the ICI levels, optimizing conventional ICI mitigation techniques implemented in MIMO-OFDM. Despite the fact that all the previous techniques can lead to a significant reduction of the noise ratio, but none of them fully satisfies the criteria of balancing complexity and efficiency.

In this research we aim to mathematically analyse the effect of $\mathrm{CP}$, and demonstrate its ability to mitigate the ICI and ISI effects in realistic wideband communication scenarios, the proposed scheme can be easily implemented because of its low complexity, simplifying the equalization at reception, while increasing significantly the signal to interference ratio. The rest of the paper is organised as followin section 2, the paper provides some basic channel models. Section 3 and 4 provide an analysis of the OFDM system. Section 5 describes the multipath effect. Section 6, presents an overview of th cyclic prefix. In section 7, we provide the simulation results in order to demonstrate the system efficiency and finally the conclusion is provided in section 8 .

\section{OFDM CHANNEL MODEL}

\subsection{AWGN channel}

AWGN Channel is the simplest channel [6] and the only parameter is the SNR [7] as shown in Figure 1.

\subsection{Rayleigh channel}

The channel impulse response of Rayleigh multipath channel could be expressed as [4], and modelled as illustrated in Figure 2 [6].

$$
h(t, \tau)=\sum_{k-1}^{L-1} U_{k} e^{j \varphi k} \delta\left(t-\tau_{k}\right)
$$

where $\mathrm{L}$ is the number of paths, $\tau_{k}$ is the delay of the $k^{t h}$ path, $U_{k} e^{j \varphi k}$ is the gain coefficient.

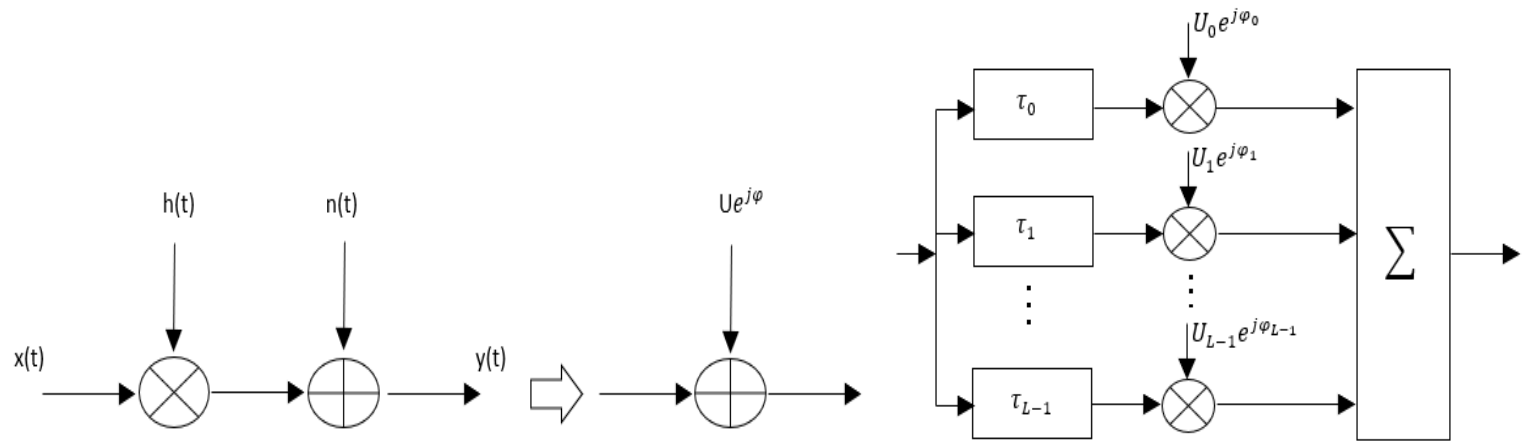

Figure 1. AWGN channel model

Figure 2. Rayleigh channel model

\section{OFDM TRANSMISSION SCHEME}

Figure 3 depicts a classical OFDM transmission scheme. The input data sequence is baseband modulated, using modulation schemes such as (BPSK, QPSK, QAM) in our system, 16 QAM method has been used in order to encode the binary information. The data symbols are converted from serial to parallel, each of the $\mathrm{N}$ parallel substream will modulate a separate carrier via the IFFT modulation block, which actually generates the OFDM symbol, performing the multicarrier modulation. The inter symbol interference and inter carrier interference are then dealt with by introducing a cyclic prefix. The cyclic prefix is obtained by copying the rear part of OFDM symbol and put it at the beginning of the symbol [8]. After Parallel to serial conversion, we can finally obtain the OFDM symbol [9]. At the receiver, the inverse operations are performed; starting with the removal of the cyclic prefix [10], the spectral decomposition of the received samples calculated using the FFT algorithm [15] and finally the demodulation [16], in the case of performing a channel estimation and additional stage is added to estimate the response of the transmission channel [17]. 


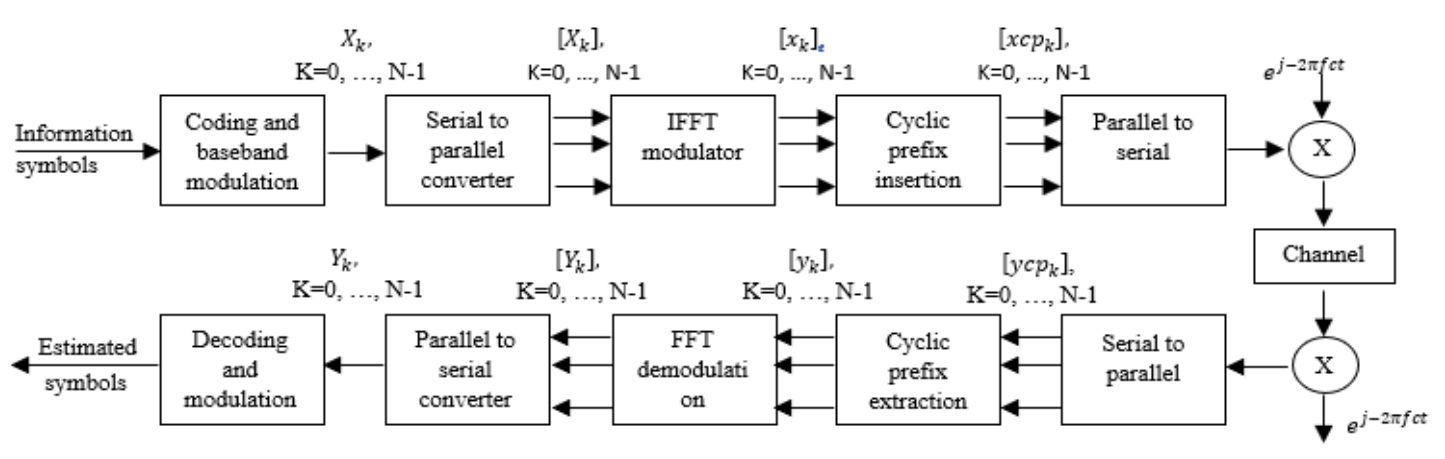

Figure 3. The block diagram of an OFDM system

\section{SYSTEM DESCRIPTION}

\subsection{Orthogonality}

Consider the time limited complex signals $\left\{e^{j 2 \pi f_{k} t}\right\}_{k=0}^{N-1}$ which represent the different subcarriers at $f_{k}=\frac{K}{T_{s y m}}$ the OFDM signal, where $0 \leq t \leq T_{\text {sym }}$. The signals are defined to be orthogonal if the integral of the products on their common (fundamental) period is zero. The orthogonality described by (1) is the main condition characteristic of an ICI free OFDM signal.

$$
\begin{aligned}
& \frac{1}{T_{\text {sym }}} \int_{0}^{T_{\text {sym }}} e^{j 2 \pi f_{k} t} e^{-j 2 \pi f_{i} t} d t=\frac{1}{T_{\text {sym }}} \int_{0}^{T_{\text {sym }}} e^{j 2 \pi \frac{k}{T_{\text {sym }}} t} e^{-j 2 \pi \frac{i}{T_{\text {sym }}} t} d t \\
& =\frac{1}{T_{\text {sym }}} \int_{0}^{T_{\text {sym }}} e^{j 2 \pi \frac{(k-i)}{T_{\text {sym }}} t} d t \\
& =\left\{\begin{array}{c}
1, \forall \text { integer } k=i, \\
0, \text { otherwise },
\end{array}\right.
\end{aligned}
$$

\subsection{OFDM modulation and demodulation}

ODFM transmitter codes the bit stream into a sequence of PSK symbols which will be subsequently parallelized into $\mathrm{N}$ streams. This conversion is carried out by different subcarriers. Let $X_{1}[k]$ denote $l^{t h}$ transmit symbol at the $k^{t h}$ subcarrier, $l=0,1,2 \ldots, \infty, k=0,1,2 \ldots, N-1$. After the serial-to-parallel conversion, the duration of transmission time for $\mathrm{N}$ symbols becomes $N T_{s}$, which forms a single OFDM symbol with a length of $T_{\text {sym }}$ (i.e., $T_{\text {sym }}=N T_{s}$ ). Let $\psi_{l . k}(t)$ be the $l^{t h}$ OFDM signal at the $k_{t h}$ subcarrier, which is given by:

$$
\psi_{l . k}(t)=\left\{\begin{array}{c}
e^{j 2 \pi f_{k}\left(t-l T_{\text {sym }}\right)} \\
0, \text { otherwise }
\end{array}\right.
$$

Then the pass band and baseband OFDM signals in the continuous-time domain can be expressed respectively as (4), (5).

$$
\begin{aligned}
& X_{1}(t)=\operatorname{Re} \frac{1}{T_{s y m}} \sum_{l=0}^{\infty} \sum_{k=0}^{\infty} X_{1}[k] \psi_{l . k}(t), \text { and } \\
& X_{1}(t)=\sum_{l=0}^{N-1} \sum_{k=0}^{N-1} X_{1}[k] e^{j 2 \pi f_{k}\left(t-l T_{s y m}\right)}
\end{aligned}
$$

The sampling of the continuous time based OFDM signal given in (4) can be carried out at $t=l T_{\text {sym }}+n T_{s}$ with $T_{s}=\frac{T_{s y m}}{N}$ and $f_{k}=\frac{k}{T_{s y m}}$ to yield the corresponding discrete-time OFDM symbol as (6).

$$
X_{1}[n]=\sum_{k=0}^{N-1} X_{1}[k] e^{j 2 \pi k n / N}, \text { for } n=0,1, \ldots . N-1
$$

Note that (6) is the N-point IDFT of PSK data symbols $x_{1}[k]_{k=0}^{N-1}$ is performed using the inverse fast Fourier transform (IFFT) algorithm. Considering the received baseband symbol $y_{1}(t)=$ $\sum_{k=0}^{N-1} X_{1}[k] e^{j 2 \pi f_{k}\left(t-l T_{s y m}\right)}, l T_{\text {sym }}<t \leq l T_{s}+n T_{s}$, the transmitted symbol $X_{1}[k]$ can be reconstructed by the orthogonality among the subcarriers in (2) as: 


$$
\begin{aligned}
& Y_{1}[k]=\frac{1}{T_{\text {sym }}} \int_{-\infty}^{\infty} y_{1}(t) e^{-j 2 \pi k f_{k}\left(t-l T_{\text {sym }}\right)} d t \\
& =\frac{1}{T_{\text {sym }}} \sum_{i=0}^{N-1} X_{1}[i] e^{j 2 \pi f_{i}\left(t-l T_{s y m}\right)} e^{-2 j \pi f_{i}\left(t-T_{\text {sym }}\right)} e^{-j 2 \pi f_{k}\left(t-l T_{\text {sym }}\right)} d t \\
& =\sum_{i=0}^{N-1} X_{1}[i] \frac{1}{T_{\text {sym }}} \int_{0}^{T_{\text {sym }}} e^{j 2 \pi\left(f_{i}-f_{k}\right)\left(t-l T_{\text {sym }}\right)} d t \\
& =X_{1}[K]
\end{aligned}
$$

where the effects of channel and noise are not considered. Let $y_{1}[n]_{n=0}^{N-1}$ be the sample values of the received OFDM symbol $y_{1}(t)$ at $t=T_{s y m}+n T_{s}$. Then, by integrating (7) in the modulation process the discrete time form of the signal is obtained:

$$
\begin{aligned}
& y_{1}[k]=\sum_{n=0}^{N-1} y_{1}[n] e^{-\frac{j 2 \pi k n}{N}} \\
& =\sum_{n=0}^{N-1}\left\{\frac{1}{N} \sum_{i=0}^{N-1} X_{1}[i] e^{\text {fracj } 2 \pi i n N}\right\} e^{-\frac{j 2 \pi k n}{N}} \\
& =\frac{1}{N} \sum_{n=0}^{N-1} \sum_{i=0}^{N-1} X_{1}[i] e^{j 2 \pi(i-k)^{n} / N} \\
& =X_{1}[k]
\end{aligned}
$$

As shown in (8) is the N-point DFT of $y_{1}[n]_{n=0}^{N-1}$ which is computed by using the fast Fourier transform (FFT) algorithm. Diagram in Figure 4 depicts the OFDM modulation and demodulation structure, for $\mathrm{N}=6$, the frequency domain symbol $X[k]$ modulates the subcarrier with a frequency of $f_{k}=\frac{k}{T_{s y m}}$, and at the other end of the reception the demodulation is performed by taking advantage of the orthogonally between subcarriers. The original symbol $[k]$ has duration of $T_{s}$, but its length has been extended to $T_{\text {sym }}=N T_{s}$ by transmitting $\mathrm{N}$ symbols in a parallel form. Figure 4 illustrates a realization of orthogonally between all subcarriers.

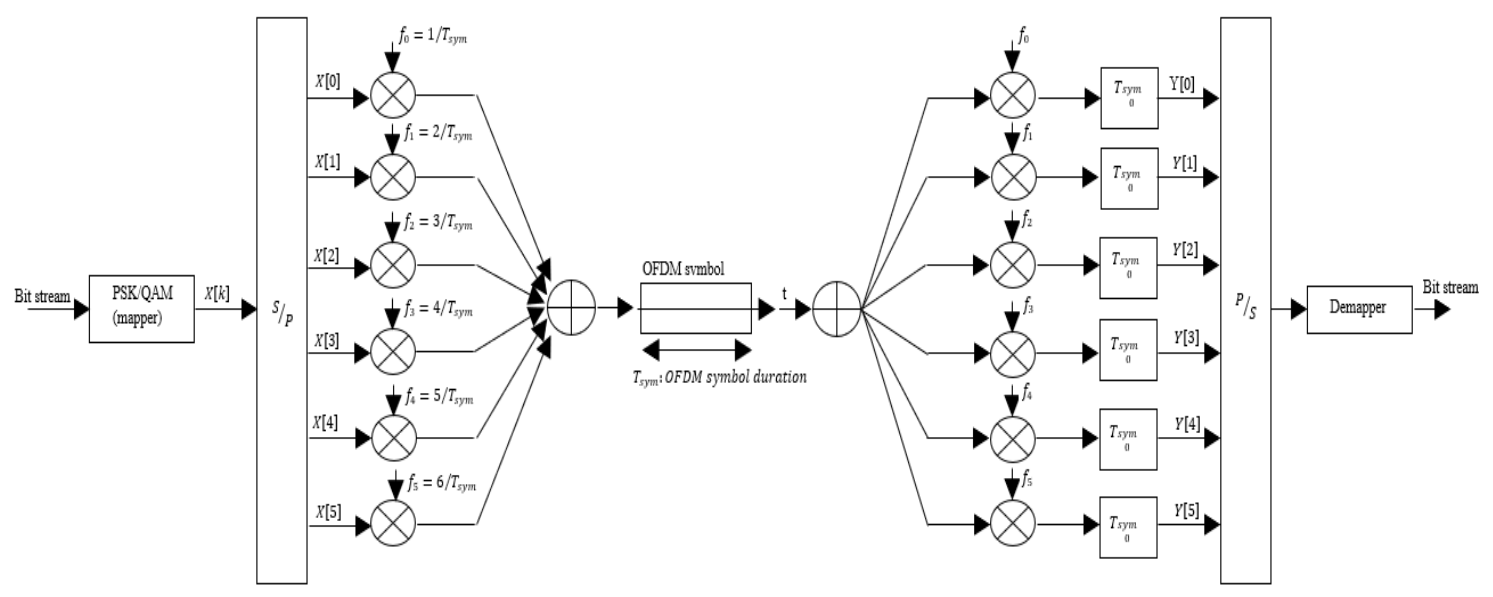

Figure 4. Block diagram of OFDM modulation and demodulation of 6 parallel streams

\section{EFFECT OF MULTIPATH}

In the following section we present the expression of SINR as a function of the following parameters: symbol length [8], CP length [18], multipath and the thermal noise [16]. The CP length is $\Delta$ seconds. The combined length of the OFDM symbol and the $\mathrm{CP}$ is $T_{G}+N T_{S}$. The function $C(\tau)$ is defined as (9). This is obtained by applying the $\mathrm{C}$ function defined in the equation and traduced in time domain as in the spectrum given in Figure 5. 


$$
C\{\tau\}=\left\{\begin{array}{cc}
0, & \tau<-N T_{S} \\
\frac{N T_{S}+\tau}{N T_{S}}, & -N T_{S}<\tau<0 \\
1, & 0<\tau<T_{G} \\
N T_{S}-\left(\tau-T_{G}\right) & T_{G}<\tau<N T_{S}+T_{G} \\
\hline N T_{S} & N T_{S}+T_{G}<\tau
\end{array}\right.
$$

In the scenario of a $\mathrm{N}$ path channels with complex gains $\alpha_{m}, h(t)=\sum_{m=1}^{N \text { paths }} \alpha_{m} \delta\left(t_{\tau m}\right)$, the SINR for a channel with transmit signal power $\sigma_{x}^{2}$ per subcarrier and thermal noise of variance $\sigma_{n}^{2}$ per subcarrier, can be written in terms of signal and interference power.

$$
\operatorname{SINR}=\frac{P_{S}}{P_{i}+\frac{\sigma_{n}^{2}}{\sigma_{n}^{2}}}
$$

where:

$$
P_{S}=\sum_{m=1}^{N_{p a t h s}} C\left(\tau_{m}\right)^{2} E\left|\alpha_{m}\right|^{2}
$$

is the useful signal power after the FFT, and:

$$
\begin{aligned}
& P_{i}=\sum_{m=1}^{N_{\text {paths }}}\left(1-C\left(\tau_{m}\right)^{2}\right) E\left|\alpha_{m}\right|^{2}=P_{h}-P_{s} \\
& P_{h}=\sum_{m=1}^{N_{\text {paths }}} E\left|\alpha_{m}\right|^{2} \text { is the channel energy. We assume that all subcarriers are occupied, only then }
\end{aligned}
$$
the expression is an approximation of the empirical ICI/ISI power, and useful power. For rays in the tapered portion of the bias function $\left(C(\tau) \leq \tau_{m}<N T_{s}+\Delta\right)$, it can be shown that their interference contribution is:

$$
\left(1-C\left(\tau_{m}\right)^{2}\right) E\left|\alpha_{m}\right|^{2}=\frac{\left(\tau_{m}-T_{G}\right)}{N T_{s}}\left[1+\frac{N T_{s}-\left(\tau_{m}-T_{G}\right)}{N T_{s}}\right] E\left|\alpha_{m}\right|^{2}
$$

In the middle term of this equation, the ' 1 ' term is from ISI, while $\frac{N T_{S}-\left(\tau_{m}-T_{G}\right)}{N T_{S}}$ term is due to ICI. For small excess delays, we can consider that the ICI term is approximately equal to the ISI term.

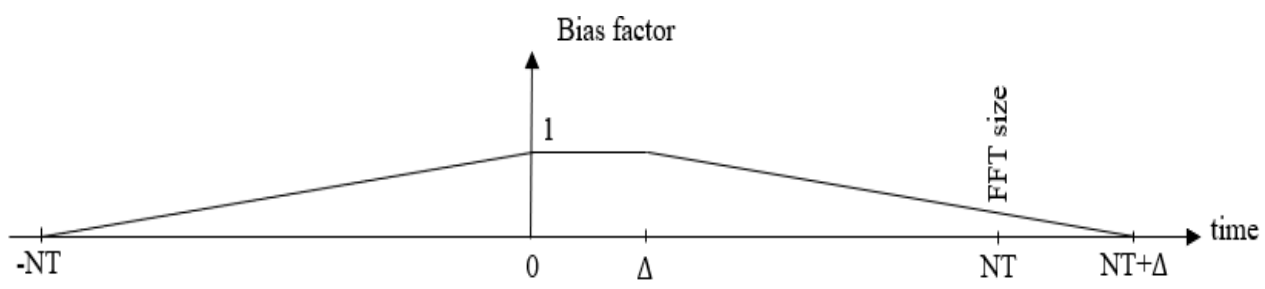

Figure 5. The function $C(\tau)$ applied to impulse response

\section{CYCLIC PREFIX}

To add a cyclic prefix is to extend the OFDM symbol by copying the last samples of the OFDM symbol into its front [19]. Let $T_{G}$ denote the length of CP in terms of samples [20] Then, the extended OFDM symbols now have the duration of $T_{\text {sym }}=T_{\text {sub }}+T_{G}$. Figure 6 shows two consecutive OFDM symbols [21], each of which has a CP of length $T_{G}$ [22]. Figure 7 illustrates the ISI generated by the multipath effect on some subcarriers of the OFDM symbol [23]. It can be seen from this figure that if the length of the cyclic prefix (CP) exceedsthe maximum delay of a multipath channel, the ISI effect of an OFDM symbol (dotted line) on the next may not affect the FFT of the next OFDM symbol, taken for the duration of $T_{\text {sub }}$ [24], this means that the guard interval must be longer than the maximum delay of the multipath channel in order to maintain the orthogonality with all other subcarriers over $T_{\text {sub }}$, such that [25]:

$$
\frac{1}{T_{\text {sub }}} \int_{0}^{T_{\text {sub }}} e^{j 2 \pi f_{k}\left(t-t_{0}\right)} d t=0, k \neq i
$$


For the first OFDM signal that arrives with a delay of $t_{0}$, and;

$$
\frac{1}{T_{s u b}} \int_{0}^{T_{s u b}} e^{j 2 \pi f_{k}\left(t-t_{0} T_{S}\right)} d t=0, k \neq i
$$

For the second OFDM signal that arrives with a delay of $t_{0}+T_{s}$.

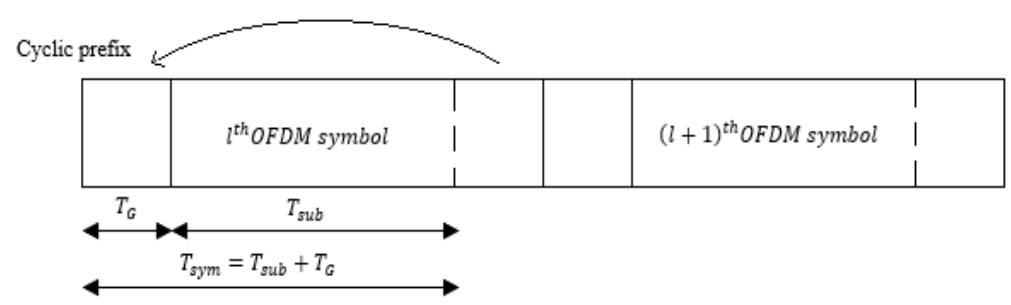

Figure 6. OFDM symbol with $\mathrm{CP}$

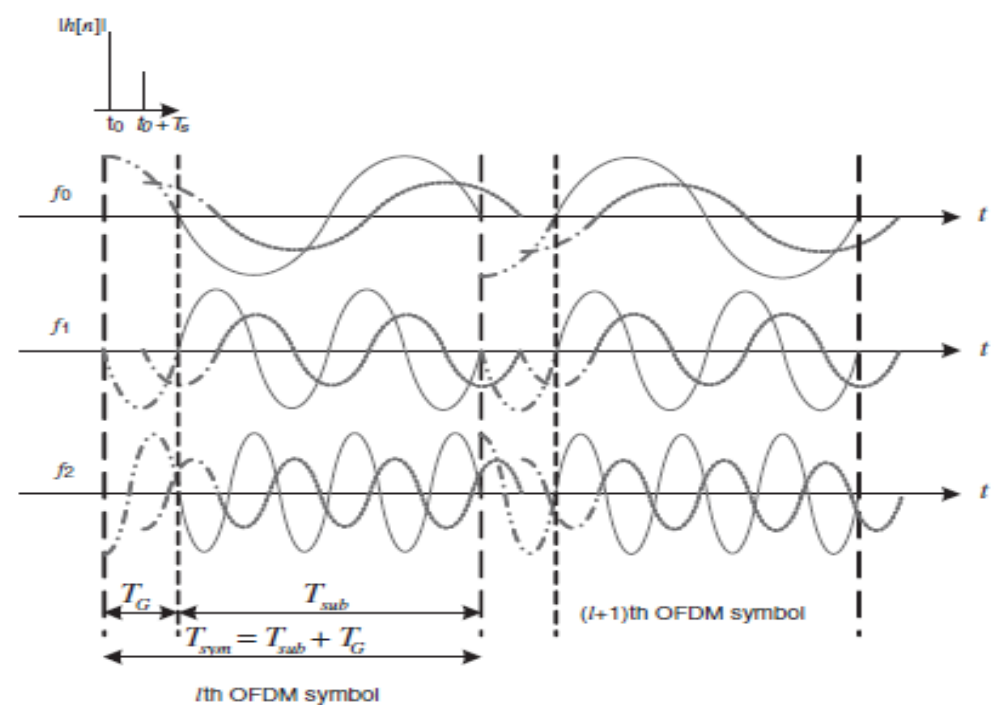

Figure 7. ISI effect of a multipath channel for each subcarrier

\section{SIMULATION AND DISCUSSION}

\subsection{Parameters of the simulation}

To simulation setup details in MATLAB commercial software are shown in Table 1, the OFDM scheme has 128 sub-carriers using 16 QAM modulation, transmitted over the AWGN and Rayleigh channel, which depends on two parameters: $\tau$ and the gain on each path.

Table 1. The parameters of the simulation

\begin{tabular}{cc}
\hline Number of subcarriers & 128 \\
Modulation scheme & $16 \mathrm{QAM}$ \\
Sampling period & $1 e^{-5}$ \\
CP length & From $0 \%$ to $30 \%$ \\
Number of frames & 1000 \\
The delay $\tau$ & $0,100 e^{-5}, 3.5 e^{-5}, 1200 e^{-5}$ \\
The gains & $0,-6,-3,-5$ \\
\hline
\end{tabular}

\subsection{Simulation}

In this work, we carried out a comparative study of the behavior of an OFDM transmission system, by varying the length of CP values from $0 \%$ to $30 \%$ of the total duration of the symbol. The metric used for this evaluation is the BER taking SNR values ranging from -5 to $20 \mathrm{~dB}$. The AWGN channel considers only 
one path between the transmitter and the receiver and only a constant attenuation noise, so no multipath effect, which makes the transmission immune to ISI. The Rayleigh fading channel instead considers multipath between transmitter and receiver as shown in Figure 8. Therefore, the communication is affected by ISI, making its BER higher than that of the AWGN channel for different values of SNR.

The introduction of the CP as shown in Figure 9, reduces the BER of the signal on the Rayleigh fading channel while the one on AWGN channel remains unchanged. This is fully consistent with the fact that the AWGN channel does not take the multipath into account, so the introduction of the CP does not improve the BER for this channel. The Rayleigh fading channel response is more affected by the CP length, because the main parameters of this channel are the frequency spread spectrum and the delay introduced by the multipath. We clearly notice that their effect is reduced by the introduction of the $\mathrm{CP}$, as shown on Figure 10 representing the BER Vs SNR for a signal without CP compared to another with a CP representing $0 \%$ to $30 \%$ from the inter symbol. We notice that keeping the signal at the same level 0.01 BER requires a $3 \mathrm{~dB}$ greater SNR or the introduction of $10 \% \mathrm{CP}$. The length of the CP needed to keep the signal at the same BER level is even more considerable when compared to the $30 \% \mathrm{CP}$ length where we need a $6 \mathrm{~dB}$ SNR, in comparison to other simple solutions, this almost matches their performance allows the SNR to grow to $45 \mathrm{~dB}$ for the blind algorithm [15], and similar performance shown when using equalization [18].

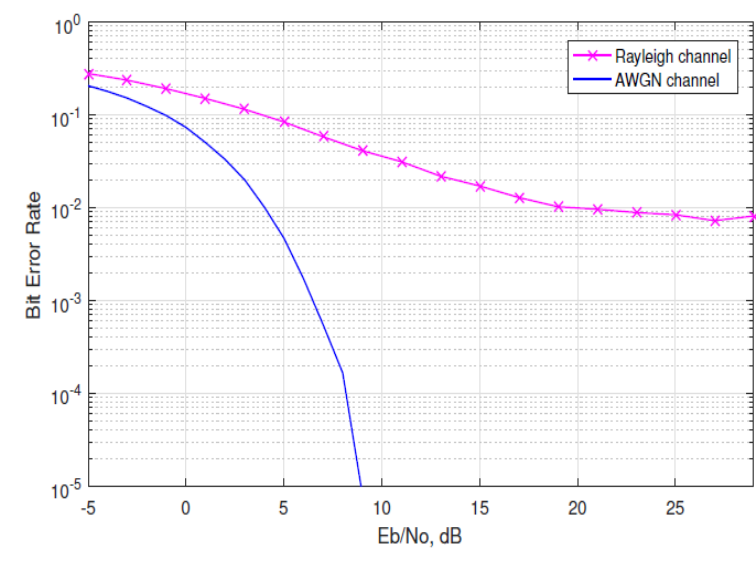

Figure 8. BER performance of OFDM system for AWGN and Rayleigh fading channel without CP

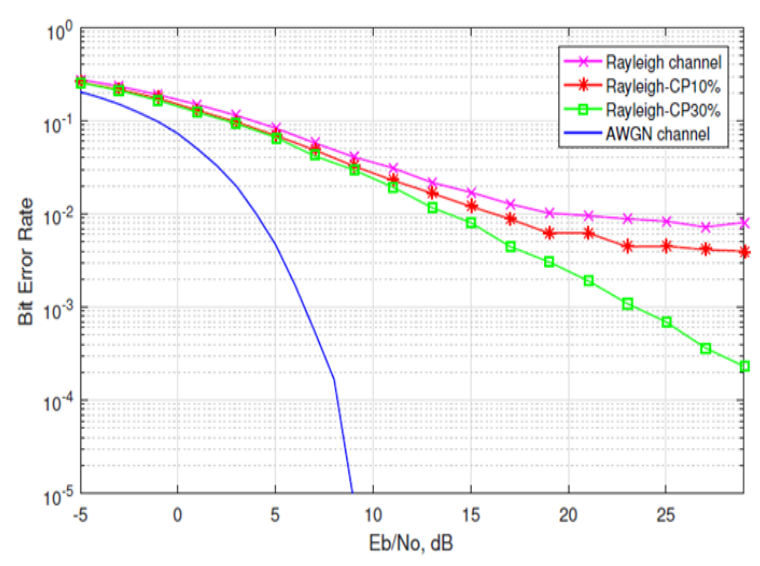

Figure 9. BER performance of OFDM system for AWGN and Rayleigh fading channel with varying CP length

\subsection{Constellation}

In the constellation diagram for the 16 QAM modulation we depict the error introduced by the channel and caused mainly by the linear distortions (amplitude ripple, group delay, low carrier-to-noise ratio) as a dispersion of the symbol points around the theoretical position, for each point of the constellation. This degradation of the quality of the modulation is given by the MER shown in (15).

$$
M E R=10 \log _{10}\left[\frac{\sum_{j=1}^{N}\left(I_{j}^{2}+Q_{i}^{2}\right)}{\sum_{j=1}^{N}\left(\delta I_{j}^{2}+\delta Q_{j}^{2}\right)}\right]
$$

Assuming that the transmitted data-symbol is represented by the coordinate pair $\left(a_{k}+b_{k}\right)$. After reception, the baseband I, Q signals are given by:

$$
\begin{aligned}
I_{k} & =\operatorname{Real}\left[\left(a_{k}+j b_{k}\right) e^{j \phi_{n}}\right] \\
& =a_{k} \cos \left(\phi_{n}\right)-b_{k} \sin \left(\phi_{n}\right)+n_{I} \\
Q_{k} & =\operatorname{Imag}\left[\left(a_{k}+j b_{k}\right) e^{j \phi_{n}}\right] \\
& =a_{k} \sin \left(\phi_{n}\right)+b_{k} \cos \left(\phi_{n}\right)+n_{Q}
\end{aligned}
$$

In the Figure 10, for the same snr value the CP length can improve the quality of the MER hence the quality of the transmission, by limiting this dispersion, and for a lower value of the SNR, the effect of the CP length is more noticeable. 


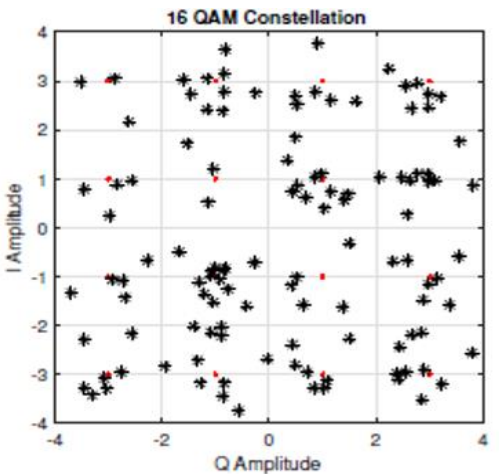

(a)

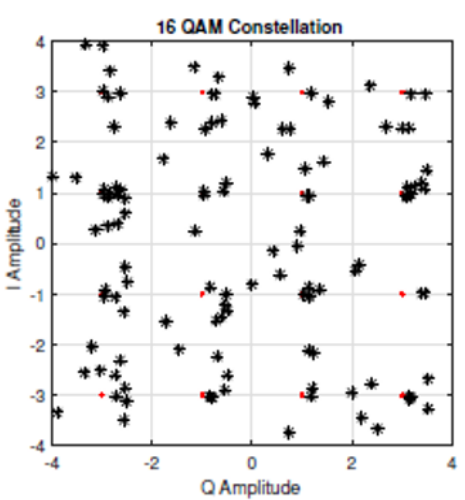

(d)

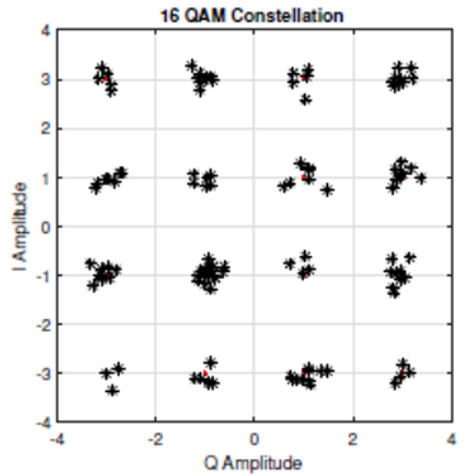

(b)

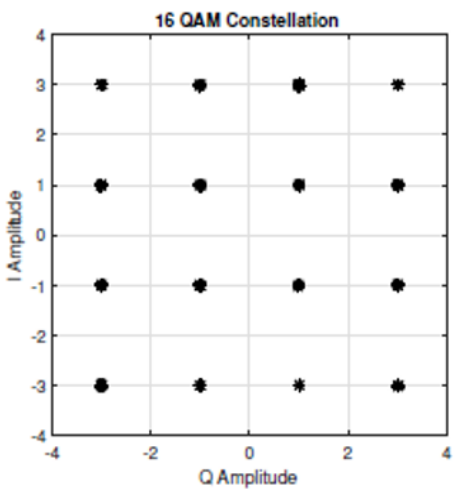

(e)

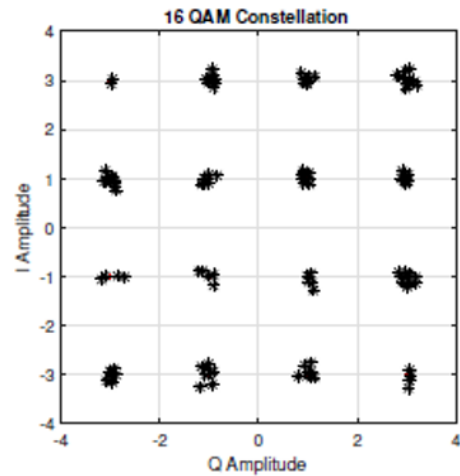

(c)

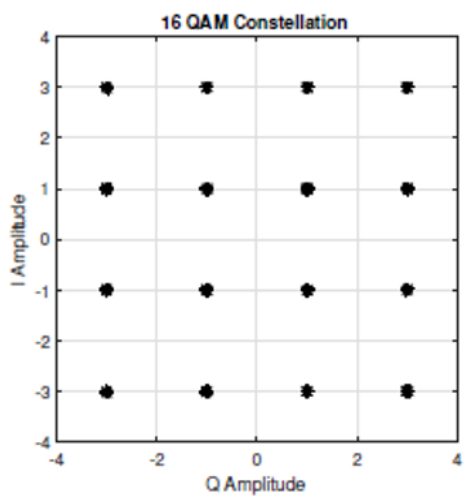

(f)

Figure 10. 16QAM constellation for two values of SNR: 20db; (a) CP 0\%, (b) CP 10\%, (c) CP 30\%, and $40 \mathrm{db}$, (d) CP 0\%, (e) CP 10\%, and (f) CP $30 \%$

\section{CONCLUSION}

In this work, we have discussed the transmission problems in the OFDM transmission scheme, the effect of $\mathrm{CP}$ length has been simulated in terms of BER for AGWN and Rayleigh fading channel. The AWGN channel considers only one path between the transmitter and receiver, whereas the Rayleigh fading channel takes into consideration the multipath which is the main reason of the received signal distortion leading to the ISI. In OFDM system this is avoided by the introduction of the CP. On the simulation we carried out, we observed that the effect of the multipath decreases with the increasing of the CP length. It would be easy to assume that the increase of the CP length has always a positive impact on the transmitted signal, but because the duration of the $\mathrm{CP}$ is deducted from the total time dedicated to the data, a trade-off must be made between the robustness of the signal and the throughput, therefore, there are limits to the size of the CP. On the other hand, we carried a simulation on the effect of the $\mathrm{CP}$ on the dispersion of the constellation in the Rayleigh channel scenario and it yields that the introduction of the $\mathrm{CP}$ could reduce it considerably.

\section{REFERENCES}

[1] Yong Soo Cho, Jaekwon, Won Young Yang, Chung-Gu Kang, "MIMO OFDM Wireless Communications with MATLAB," Wiley, 2010.

[2] Z. Sheng, H. D. Tuan, H. H. Nguyen and Y. Fang, "Pilot Optimization for Estimation of High-Mobility OFDM Channels," IEEE Transactions on Vehicular Technology, vol. 66, no. 10, pp. 8795-8806, 2017.

[3] P. T. Agarkar, N. G. Narole, P. R. Hajare and N. G. Bawane, "A Novel LS-LMMSE Channel Parameter Tuning Approach using Particle Swarm Optimization in MIMO-OFDM," 2018 International Conference on Current Trends towards Converging Technologies (ICCTCT), Coimbatore, India, 2018, pp. 1-6.

[4] H. Rohling, "OFDM, Concepts for Futur Communication Systems," Springer-Verlag Berlin Heidelberg, 2011.

[5] S. Hara, R. Prasad, "Multicarrier Techniques for 4G Mobile Communications," Artech House, 2003, Art. no. 268.

[6] E. Kadhum, R. Haitam, "Performance Analysis of IEEE 802.15.4 Transceiver System under Adaptive White Gaussian," International Journal of Electrical and Computer Engineering (IJECE), vol. 8, no. 6, pp. 4184-4196, 2018.

[7] M. I. Youssef, A.E. Emam, M. Abd Elghany, "ICI PAPR enhancement in MIMO-OFDM system using RNS coding," International Journal of Electrical and Computer Engineering (IJECE), vol. 9, no. 2, pp. 1209-1219, 2019. 
[8] Iskandar and W. Khabzli, "Comparison of preamble and Cyclic Prefix method for Frequency Synchronization in OFDM systems," 2016 10th International Conference on Telecommunication Systems Services and Applications (TSSA), Denpasar, 2016, pp. 1-5.

[9] J. Lee and H. Ryu, "Design and Comparison of Discrete Wavelet Transform Based OFDM (DWT-OFDM) System," 2018 Tenth International Conference on Ubiquitous and Future Networks (ICUFN), Prague, Czech Republic, 2018, pp. 881-885.

[10] B. Sheng, "Non-Data-Aided Measurement of Noise Variance for OFDM System in Frequency-Selective Channels," IEEE Transactions on Vehicular Technology, vol. 65, no. 12, pp. 10184-10188, 2016.

[11] Iskandar, I. Setyawan, and H. Nuraini, "Inter-cell Interference Management Technique for Multi-Cell LTE-A Network," International Journal of Electrical and Computer Engineering (IJECE), vol. 7, no. 5, pp. 2696-2705, 2017.

[12] A. S. Bedi, J. Akhtar, K. Rajawat and A. K. Jagannatham, "BER-Optimized Precoders for OFDM Systems with Insufficient Cyclic Prefix," IEEE Communications Letters, vol. 20, no. 2, pp. 280-283, 2016.

[13] V. K. Gupta and S. Vijay, "A Summative Comparison of Blind Channel Estimation Techniques for Orthogonal Frequency Division Multiplexing Systems," International Journal of Electrical and Computer Engineering (IJECE), vol. 8, no. 5, pp. 2744-2752, 2018.

[14] M. A. Khalifa, A. E. Emam And M. I. Youssef, "ICI and PAPR Enhancement in MIMO-OFDM System Using RNS Coding," 2019 IEEE Jordan International Joint Conference on Electrical Engineering and Information Technology (JEEIT), Amman, Jordan, 2019, pp. 7-12.

[15] M.Batariere, K. Baum, T. P. Krauss, "Cyclic Prefix Length Analysis for 4G OFDM Systems," IEEE 60th Vehicular Technology Conference, 2004. VTC2004-Fall. 2004, Los Angeles, CA, USA, vol. 1, 2004, pp. 543-547.

[16] S. Ghazi-Maghrebi, H. Motahayeri, K. Der Avanesian, M. Lotfizad, "A New Mathematical Analysis of the Cyclic Prefix Effect on Removing ISI and ICI in DMT Systems," TENCON 2011-2011 IEEE Region 10 Conference, Bali, Indonesia, 2011, pp. 237-241.

[17] M.Oltean and M. Nafornita, "The Cyclic Prefix Length Influence on OFDM-Transmission BER," Buletinul Atiin al University 'Politehnica' din TimiAoara, 2003.

[18] B. Bhattacharryya, I. Saha Misra, S. K. Sanyal, "The Effect of varying Cyclic Prefix on Residual Constellation Error in OFDM Technology Using a Novel Simulink-VSA Based WiMAX Transceiver," Proceedings of Papers 5th European Conference on Circuits and Systems for Communications (ECCSC'10), 2010, pp. 268-271.

[19] A. Al-jzari, I. Kostanic, H. Hassan Mohamed Mabrok, "Effect of Variable Cyclic length on OFDM System Performance over Different Wirless Channel Models," Univ. J. of Commu. and Net., vol. 3, no. 1, pp. 7-14, 2015.

[20] Prafulla D. Gawande, Sirddharth A. Ladhake, "BER Performance of OFDM System withCyclic Prefix and Zero Padding," International Journal of Advances in Engineering and Technology, vol. 6, no. 1, pp. 316-324, 2013.

[21] Amber L. Scott, "Effect of Cyclic prefix Jamming Versus Noise Jamming in OFDM Signals," Air Force Institute of Technology, Department of the Air Force Air University, 2011.

[22] Payaswini P. and Manjaiah D. H., "Analysis of Effect of Cyclic Prefix on Data Rates in OFDM Modulation Techniques," International Journal of Advanced Computer and Mathematical Sciences, vol. 3, no. 4, pp. 465-470, 2012.

[23] I. A. Hieder, "Improvement of Fading Channel Modeling Performance for Wireless Channel," International Journal of Electrical and Computer Engineering (IJECE), vol. 8, no. 3, pp. 1451-1459, 2018

[24] A. Deshmukh and S. Bodhe, "Comparison of DCT and Wavelet Based OFDM System Working in 60 GHz Band," International Journal of Advancements in Technology, vol. 3, no. 2, pp. 74-83, 2012.

[25] H. Wu, J. Li, B. Dai and Y. Liu, "Analysis of the Impact of AGC on Cyclic Prefix Length for OFDM Systems," IEEE Transactions on Communications, vol. 66, no. 10, pp. 4783-4794, 2018.

\section{BIBLIOGRAPHY OF AUTHORS}

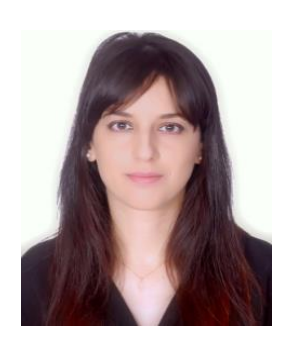

Sarah Zanafi, received the license in Electronics Electrotechnics and Automatics (EEA) from Abdlmelek Essaidi university, faculty of science and Technology, Tangier, Morocco in 2011 and the Master degree in telecommunication systems engineering (TSE) from faculty of science, Tetuan in November 2013 and is currently pursuing the Ph.D. degree. His research interests are new-generation mobile system design, radio network planning and optimization.

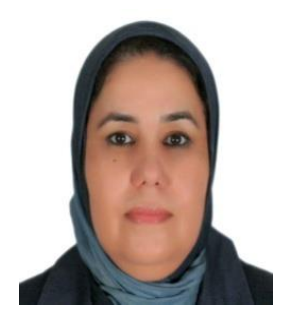

Noura Aknin, Professor of Electrical \& Computer Engineering at Abdelmalek Essaadi University since 2000. She received PhD degree in Electrical Engineering in 1998. She is the Head of Research Unit Information Technology and Modeling Systems. She is R\&D project manager/member related to new technologies and their applications. She was a chair of several conferences and she has been involved in the organizing and in the Scientific Committees of several international conferences held worldwide dealing with Information and Communication technologies. Her research interests focus mainly on mobile and wireless communications, ICT and their applications. She has authored and co-authored several papers covering different topics related with the fields cited above. 